\title{
THE FACETS OF A PROGRESSIVE RESEARCH FACILITY - CENTRAL INTER-DISCIPLINARY RESEARCH FACILITY (CIDRF)
}

\author{
Balanehru S, Deputy Director \\ Adithan C, Director \\ Central Inter-Disciplinary Research Facility
}

Sri Balaji Vidyapeeth Mahatma Gandhi Medical College and Research Institute Campus

Pillaiyarkuppam, Puducherry - 607403, India

Email: director@cidrf.res.in

i

CIDRF was established as an inventive and independent research institute under the Sri Balaji Educational and Charitable Public Trust of Puducherry. It was inaugurated on October 29, 2012 by Dr. S.R. Rao, Advisor, Dept. of Biotechnology, Ministry of Science and Technology, Government of India, New Delhi.

CIDRF has a state-of-the-art biomedical research facility. It has modern and modular infrastructure; unique and first of its kind in Pondicherry and this part of Tamilnadu. The uniqueness lies in the conception and organization of laboratories, all of which are clean rooms with totally controlled and regulated airflow; temperature and pressure. Of these laboratories, the culture laboratories (two in number with Class II and Class III bio-safety cabinets) meant to culture stem cells and other cell lines is Class 1000 while the other laboratories are Class 10,000 and the corridor, as well as common areas are Class 1lakh in international standards of air purity. The Servo-stabilized inverter backed up power is made available around the clock. Smoke and fire detectors and other safety measures are in place. A walk in cold room maintained at $4^{\circ} \mathrm{C}$ exclusively for large scale media storage and column separation etc., biology laboratory with class 10,000 area is kept away from other labs to avoid contamination. There is a media kitchen and store with specially ventilated cupboards for organic solvents and acids separately, minus 80 and minus $20^{\circ} \mathrm{C}$ storage cabinets etc. There is an emergency shower for face, eye and body wash in case of accidents. There are very strict and specific protocols to be followed by workers and even visitors in CIDRF.

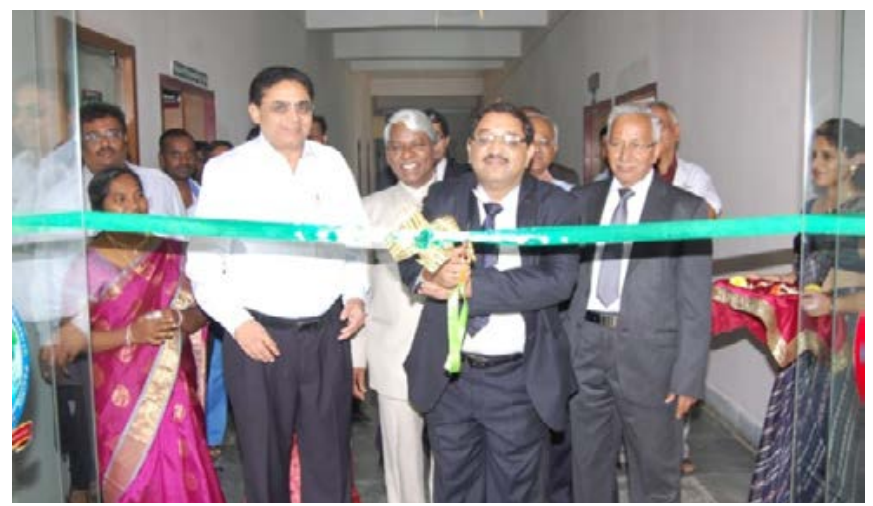

CIDRF has a defined clear vision and mission:

Vision:

- Leadership in Translational Personalized Healthcare Research

Mission:

- Expertise in cutting edge research

- Increased impact on patient care

- Expanded scope of clinical practice

- Institutional knowledge coordination

- Knowledge-base in healthcare research

CIDRF bridges and coordinates the research interests of medical, dental, nursing and scientific faculty under Sri Balaji Vidyapeeth and beyond. Research projects that originate with pre-, para- and clinical researchers are jointly developed by CIDRF in a consortium style of functioning sharing mutual strengths and 
resources available at Mahatma Gandhi Medical College \& Research Institute, Shri Sathya Sai Medical College and Research Institute, Indira Gandhi Institute of Dental Sciences, Kasturiba Gandhi Nursing College and external collaborators. CIDRF unites the forces of government, academia and entrepreneurial entities to promote and support biomedical research and healthcare. The corporal outcomes will include new medicines, novel diagnostics and new technologies that advance biomedical research and patient care resulting in stronger and larger network of "Centers of Biomedical Excellence" in India.

PRIDE is the acronym for the thrust areas of research of CIDRF where,

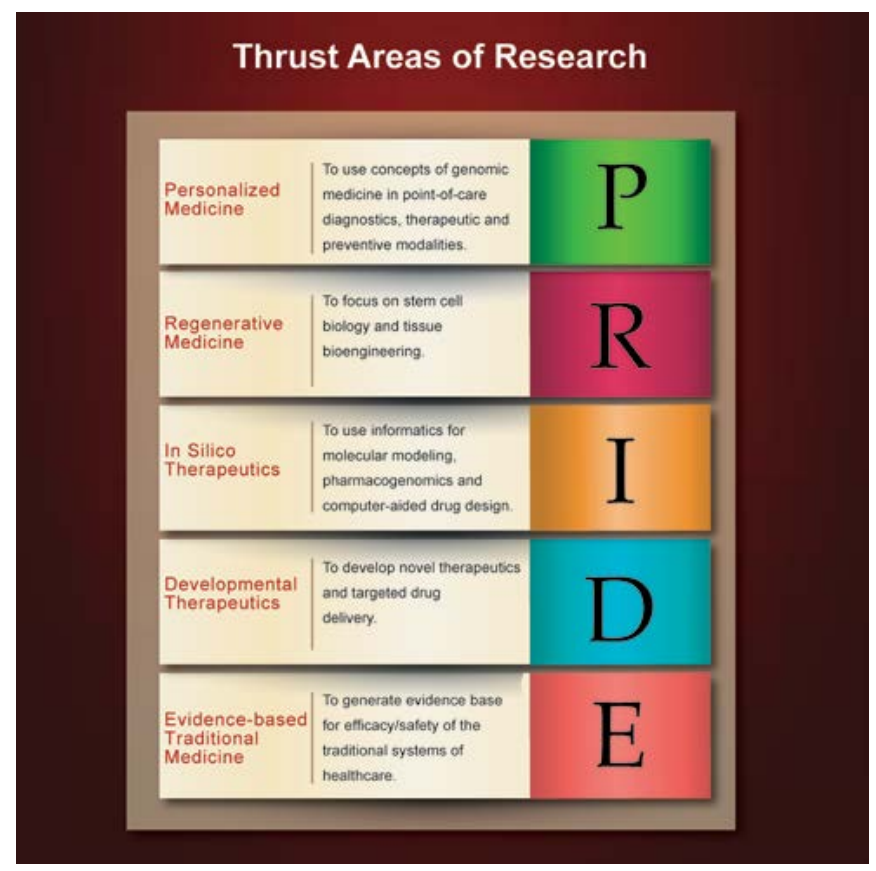

\section{Research activities}

- Since its inception, CIDR's research activities involved securing 6 extramural research grants from Government of India and Government of Puducherry.

- Nine intramural research grants from and Sri Balaji Vidyapeeth Research Fund.

- This is in addition to the 10 inter-disciplinary clinical research projects established in collaboration with clinicians of MGMCRI, SSSMCRI and IGIDS.
- Nine Research collaborations with both national/international, academic/industry have been established so far.

- In-house research activities till date has resulted in 21 publications and filing of three provisional patents with Government of India.

- Doctoral degree program affiliated to Sri Balaji Vidyapeeth has enrolled seven Ph.D. internal/ external candidates.

- An undergraduate medical student research preceptorship program was established in 2014.

- Research mentorship to both medical/dental PG students and faculty is active.

- CIDRF has so far conducted 4 conference/ seminar/workshop as part of its knowledge dissemination program.

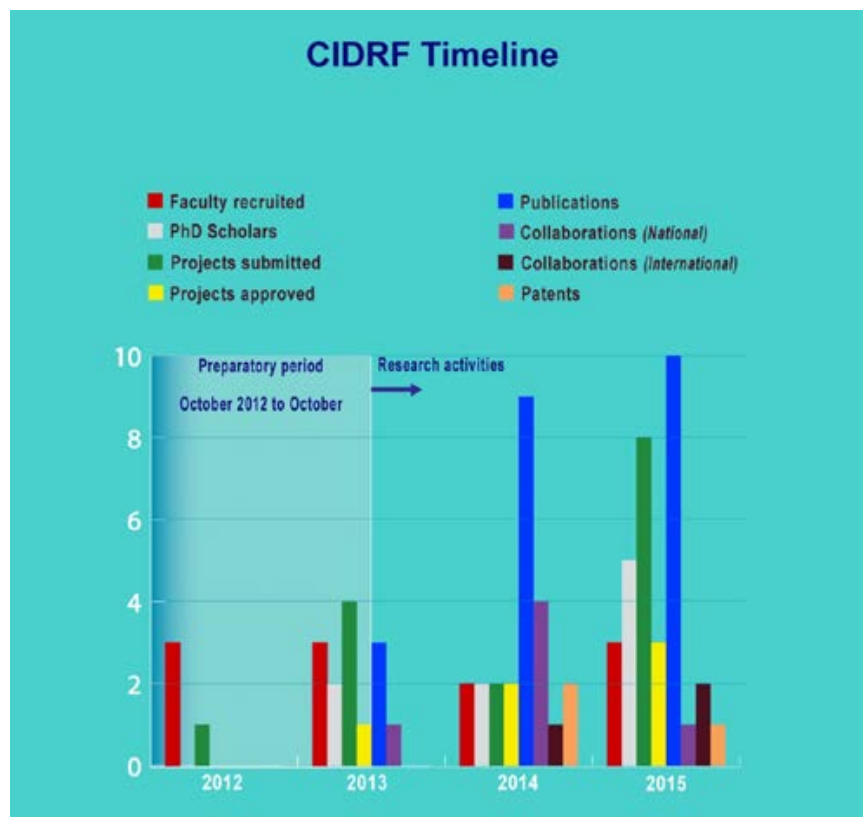

\section{Innovative programs}

1. With the aim of training interested postgraduates to gain expertise in fundamentals of Pharmacogenomics and Personalized Medicine, CIDRF is starting a one year PG Diploma program under the umbrella of CIDRF-Innovative PG Diploma programs.

2. With the aim of training interested postgraduates to be sound in fundamentals of 
modern Bio-Medical / Life Science research, CIDRF proposes following programs under the umbrella of Central Inter Disciplinary Research Facility's Innovative Certificate and Fellowship Program in Bio-Medical Research Techniques (CIDRF-IceFeP BMRT) :

a) Certificate Program in Bio-Medical Research Techniques (Cert.in BMRT) - Three Months (One trimester)

b) Fellowship Program in Bio-Medical Research Techniques (FBMRT) - One Year (Four trimesters)

\section{CIDRF-DBT Center for Animal}

Research, Training and Services (CAReTS, pronounced as carrets)

CIDRF has teamed up with DBT, Government of India to establish a new state of art small animal research facility to serve the community. This new research facility designed with modern infrastructure and latest tools would result in the establishment of a GLP compliant pathogen free animal housing and a state of art small animal research facility first of its kind, in this part of the country. This will be engaged in preclinical research, services dedicated for safety assessment, toxicology and development of medical therapeutics and devices. This new facility will serve the $\mathrm{R} \& \mathrm{D}$ needs of researchers in both public and private sectors in and around Pondicherry as well as Central and Southern Tamilnadu. Pondicherry has more than thirty biotechnology and pharmaceutical companies and twelve medical and life sciences research institutions. However, the availability and accessibility of Specific Pathogen Free (SPF) animals for quality and advanced animal research in preclinical studies and therapeutics has been a great limitation. This gap will now be filled for the benefit of users. This state of art specific pathogen free animal facility is therefore a unique research initiative to serve this part of the country.

The proposed small animal facility also proposes to provide medium to high skill development courses for qualified low-skilled technical manpower, improved quality of medical/surgical education, and increase employment opportunities. These courses will have 3 months and 6 months duration including a period for internship exposure to all the techniques in the new facility.
Yet another unique objective of the new facility would be to make available to needy scientists, knock-out animals and indigenous disease models that can be valuable for understanding the etiology of disease or for testing potential therapies specific to Indian population.

The new facility will house laboratories dedicated to:

- Small animal (rat and mice) housing and testing area

- Tissue Processing

- Cell Culture Research

- Molecular Biology

- Toxicology, Histology/Pathology

- Biomaterials \& Bioengineering

The new small animal facility will be custom designed and built featuring latest laboratory concepts and systems equipped with advanced equipment; allowing regional researchers to gain global recognition.

\section{Personalized Genetic Testing Service}

Pharmacogenomics and personalized medicine promises to markedly improve the individualized drug therapy. Realizing its importance and clinical utility, CIDRF, for the first time in Pondicherry, is introducing genetic testing of CYP2D6, CYP2C9, CYP2C19, VKORC1 and P2Y12 polymorphic genes for patient care services. The results of these tests will help to tailor the posology of anti-platelet drugs, anti-epileptics, anticoagulants, anticancer drugs, beta blockers and other commonly used drugs. This technological advancement of CIDRF will shortly be introduced at MGMCRI and to other needy hospitals.

\section{Milestones for next five years:}

1. Develop a nutraceutical product based on the research outcomes of DBT funded project entitled "Prophylactic potential of the extract of the bark of Terminalia arjuna (Roxb.ex.Dc.) Wight \& Arn. in preventing osteoporosis".

2. Establish and extend PCR based testing, on demand, for $H$. pylori treatment

3. Add Pharmacogenetics of cardio-protective drugs and anti-psychotic drugs to the newly established personalized genetic testing services. 
4. Establish research programs on MRSA and Salivary biomarkers.

5. Secure Early Career Research Awards from National and International agencies for Scientists of CIDRF.

In order to achieve our research objectives, the available research facility will be extended to accommodate a state-of-art

1. Pharmacogenomics Lab.

2. Proteomics Lab
3. Regenerative Medicine Lab.

4. Bio-informatics Lab

5. Bio-analytical Lab

6. Microbiome Lab.

7. Natural Products Lab and

8. Molecular virology Lab

9.

With a defined roadmap, we hope the facets of CIDRF in research; human resource development, new facility and lab space development, innovative academic activities and unique patient care services are well set to be the progressive inter-disciplinary research 